\title{
Long-term biochemical results after high-dose- rate intensity modulated brachytherapy with external beam radiotherapy for high risk prostate cancer
}

Pedro J Prada ${ }^{1,3^{*}}$, Lucia Mendez ${ }^{1}$, José Fernández², Herminio González ${ }^{1}$, Isabel Jiménez ${ }^{1}$ and Elisabeth Arrojo ${ }^{1}$

\begin{abstract}
Background: Biochemical control from series in which radical prostatectomy is performed for patients with unfavorable prostate cancer and/or low dose external beam radiation therapy are given remains suboptimal. The treatment regimen of HDR brachytherapy and external beam radiotherapy is a safe and very effective treatment for patients with high risk localized prostate cancer with excellent biochemical control and low toxicity.
\end{abstract}

\section{Introduction}

Patients with clinical stage T1c, Gleason score sum 6 tumors, and prostate-specific antigen (PSA) values $<10$ $\mathrm{ng} / \mathrm{ml}$ have a high likelihood of disease-free survival, regardless of the treatment option chosen.

Nevertheless, we are faced with a great dilemma when we seen newly diagnosed patients with high-risk prostate cancer. Unfortunately, results of conventionally accepted therapies such as radical prostatectomy and/or standard radiotherapy have not provided these patients with good outcomes [1-3].

The need for increased dose in patients with prostate cancer was suggested by dose response observations by Pollack $[4,5]$ and Hanks [6,7].

In an effort to improve outcomes several new radiation therapy strategies have been developed over the last decade. One approach was a combination of external beam radiotherapy (EBRT) with an intensity modulated with high dose rate (HDR) prostate brachytherapy boost.

It has been possible to increase dose, thanks to the brachytherapy advances, which allows for an increase in tumour dose (boost doses greater than 125 Gy can be safely delivered) while reducing the volume of surrounding normal tissue that is irradiated.

\footnotetext{
* Correspondence: pprada@telecable.es

'Department of Radiation Oncology, Hospital Universitario Central de

Asturias, Oviedo, Spain

Full list of author information is available at the end of the article
}

From a biologic perspective, the low prostate cancer $\alpha / \beta$ [8] favors a large dose per fraction in terms of cancer control. At the same time, the $\alpha / \beta$ of the rectum and bladder favors larger doses per fraction to increase the therapeutic window, thus improving control while limiting toxicity.

This study reports the long-term outcome during the PSA era for patients with high-risk prostate cancer who were treated with intensity modulated HDR boost.

\section{Material and methods}

Selection of patients

From June 1998 to August 2006, 252 consecutive patients were treated for high risk clinically localized prostate cancer with external beam radiation and intensity modulated HDR brachytherapy boost.

\section{Staging}

In all cases, staging evaluation included a history and physical examination, digital rectal palpation, serum PSA, chest X-ray, bone scan, abdominal CT and/or MRI, transrectal ultrasound (TRUS), and TRUS-guided biopsy with Gleason score histologic grading. All patients were $x^{\prime}$ according to the American Joint Committee on Cancer (AJCC) 4th edition [9]. Tumour characteristics are shown in Table 1. 
Table 1 Patient and tumor characteristic $(n=252)$

\begin{tabular}{|c|c|}
\hline Characteristics & $\mathrm{N}^{\circ}$ Patients (\%) \\
\hline \multicolumn{2}{|l|}{ Stage } \\
\hline$\leq \mathrm{T} 2 \mathrm{a}$ & $36(14 \%)$ \\
\hline $\mathrm{T} 2 \mathrm{~b}$ & $58(23 \%)$ \\
\hline$\geq \mathrm{T} 2 \mathrm{C}$ & $158(63 \%)$ \\
\hline \multicolumn{2}{|l|}{ Geason score: } \\
\hline$\leq 6$ & $109(43 \%)$ \\
\hline$=7$ & 77 (31\%) \\
\hline$>7$ & $66(26 \%)$ \\
\hline \multicolumn{2}{|l|}{ Pretreatment PSA (ng/ml) } \\
\hline$\leq 10$ & $44(17 \%)$ \\
\hline $10.1-20$ & $101(40 \%)$ \\
\hline$>20$ & $107(43 \%)$ \\
\hline \multicolumn{2}{|l|}{ Mean:20/Median 18 (2.05-59.60) } \\
\hline \multicolumn{2}{|l|}{ Adjuvant hormonal ablation } \\
\hline Yes & $173(69 \%)$ \\
\hline No & 79 (31\%) \\
\hline \multicolumn{2}{|l|}{ Age at diagnosis (yr) } \\
\hline$\leq 60$ & $32(13 \%)$ \\
\hline $61-70$ & $131(52 \%)$ \\
\hline$>70$ & 89 (35\%) \\
\hline \multicolumn{2}{|l|}{ Risk Level } \\
\hline High Risk by Gleason y/o PSA & $187(74 \%)$ \\
\hline High Risk by $T$ & $65(26 \%)$ \\
\hline \multicolumn{2}{|l|}{ No. Prognostic factors } \\
\hline 2 intermediate Risk Criteria & $44(17 \%)$ \\
\hline 1 High Risk Criteria & $100(40 \%)$ \\
\hline 2 High Risk Criteria & $89(35 \%)$ \\
\hline$>2$ Risk Criteria & $19(8 \%)$ \\
\hline Gland Vol. Implant (cc): Mean:34/Me & \\
\hline
\end{tabular}

Excluded from the program were those patients who had any of the following conditions:

- Previous radiotherapy to the pelvis

- Patients with another malignant process (except skin tumour) 5 years before the diagnosis of the prostate cancer

- Recurrence of prostate tumour

- Life expectancy $<5$ years

\section{Definition groups}

Patients were considered high risk according to the Memorial Sloan Kettering group definition (clinical stage $\geq$ T2c or prostate-specific antigen, PSA $>20 \mathrm{ng} /$ $\mathrm{ml}$ or Gleason score $>7$ or 2-3 intermediate-risk criteria) [10].

\section{Hormonal therapy}

In our patient population, more than half of the patients, 69\% received hormonal ablative treatment
(LhRh agonists + antiandrogens) for one year. It was initiated as neo-adyuvant treatment, three months before the start of radiotherapy.

\section{Treatment}

For several years, patients diagnosed with prostate cancer have been treated at our Institution with EBRT interdigitated with two intensity modulated brachytherapy (IMBT) HDR boosts (Table 2).

Total pelvic external beam radiation technique was 46 Gy delivered in 23 fractions of 2 Gy over 4.5 weeks. All patients were treated using 18-MV photons. No external radiation was delivered the same day of the HDR brachytherapy procedure (day 5 and 15). Total treatment time including the HDR boost was over a period of 5 weeks. All fields were treated daily. Isocentric technique was used and all fields were equally weighted. The portals used covered the prostate, seminal vesicles, and the periprostatic tissues with a margin of at least $1 \mathrm{~cm}$. The tumour volume was encompassed in the fields by the $100 \%$ isodose line $\pm 5 \%$.

Brachytherapy procedures were done under spinal anesthesia. The dose administered in each application was $11.5 \mathrm{~Gy}$, except in the first group of patients treated (17\%) in whom the dose applied varied between 10.5 and 11. The target volume of the implant was the prostate gland $+5 \mathrm{~mm}$ peri-prostatic area and medial aspects of seminal vesicle. All patients were discharged from the center the same day of the procedure between 6-8 hours of implantation.

The total combined BED dose ranged from 292 Gy to 366 Gy based upon a $\alpha / \beta$ ratio of 1.2 [8].

\section{Toxicity}

Patients were followed with symptom assessment and PSA determinations every 3 months for the first year, every 6 months for the second year and yearly thereafter.

Toxicity was reported according to the Common Toxicity Criteria for Adverse Event, Version 4.0 (CTAE v4.02) by the National Cancer Institute. Chronic toxicity was defined as those symptoms which persisted or presented beyond 6 months. Potency was defined as the

\section{Table 2 Treatment scheme}

\begin{tabular}{lll}
\hline Weeks & External radiotherapy & Brachytherapy \\
\hline 1st week & 2 Gy/day $\times 4$ days & 1st HDR; 5th day (11.5 Gy) \\
2nd week & 2 Gy/day $\times 5$ days & No \\
3rd week & 2 Gy/day $\times 4$ days & 2nd HDR; 15th day (11.5 Gy) \\
4th week & 2 Gy/day $\times 5$ days & No \\
5th week & 2 Gy/day $\times 5$ days & No \\
Total dose & 46 Gy/23 sessions & 23 Gy/2 sessions \\
\hline
\end{tabular}

HDR = high dose rate (brachytherapy) 
ability to achieve an erection that was sufficient for intercourse.

Toxicity and sexual side-effects was scored by the physician.

\section{Statistical considerations}

Distant metastatic disease was defined by an imaging study or physical examination that demonstrated cancer outside of the prostate and its regional nodes. Failure in cause-specific survival (CSS) analyses was defined as death due to prostate cancer. Failure in tumour-free survival (TFS) analyses was represented as detection of local and/or systemic tumour relapse, and bNED for no clinical and biochemical evidence of disease. Overall survival reflected all deaths, cancerrelated or otherwise. To assess the local relapse, sextant prostate biopsies were taken in patients with no metastatic disease if they did not refuse this procedure. Biochemical failure was defined according to the "Phoenix definition" [11] consensus panel statement. Estimated likelihood of events was calculated by the Kaplan-Meier method from the time of completion of radiotherapy. The statistical significance of the difference between estimated event-free

Curves were calculated with the long-rank test. Multivariate analysis was performed using the Cox proportional hazards model [12]. Statistical analyses were performed with SPSS version 17.0 (SPSS Inc, Chicago, IL).

\section{Results}

\section{Clinical characteristics}

All patients treated in the protocol were included for analysis and completed the planned course of radiation. All patients have been seen in follow up.

The high risk group, as defined in this study, represented $100 \%$ of the patient population (74\% were high risk by Gleason and/or PSA and $26 \%$ by T-stage) (Table 1).

The median V100, V90, V150 and V200 (\% volume of CTV receiving $100 \%$ of prescription dose) were respectively $93.73 \%$ (96.82-85.81), 97.90\% (99.80-91.79\%), 21.02\% (38.17-12.64) and 5.97\% (9.41-3.30). The median D90 (The dose that covers $90 \%$ volume of CTV) was 12.04 Gy (18.38-9.45). Maximum urethral point dose was 12.66 Gy (16.36 Gy-9.25 Gy) and maximum rectal point dose was $9.56 \mathrm{~Gy}$ (14.09 Gy-8.2 Gy).

\section{Oncologic endpoints}

Of all 252 patients, 51 had evidence of biochemical relapse, 42 had clinical relapse and 12 died from prostate cancer; 35 patients died of other illnesses. Mean and median follow-up for all patients were 77 and 74 months respectively with a range of 12 to 142 months.
In the 51 patients with a biochemical failure, the median time to PSA failure was 38 (5-80) months, with 55\% failing within 3 years and 98\% within 6 years. In patients with no biochemical failure, the mean and median PSA level after treatment was 0.10 and $0.03(0.0-1.2) \mathrm{ng} / \mathrm{ml}$, the last follow-up PSA levels were $\leq 0.2 \mathrm{ng} / \mathrm{ml}$ in $90 \%$, < $1 \mathrm{ng} / \mathrm{ml}$ in $99.6 \%$ and $1.2 \mathrm{ng} / \mathrm{ml}$ in a patient.

The 5 and 10 years for biochemical control were 84\% and $78 \%(\mathrm{SD} \pm 2 \%$ ), whereas in tumor-free survival (TFS) they were $86 \%$ and $82 \%(\mathrm{SD} \pm 2 \%)$ at 5 and 10 years respectively. The 10 year cause specific survival was $93 \%(\mathrm{SD} \pm 2 \%)$ with $99 \%(\mathrm{SD} \pm 1 \%)$ of patients being free of local recurrence. The overall survival according to Kaplan-Meier estimates was $88 \%$ and $78 \%$ $(\mathrm{SD} \pm 4 \%)$ at 5 and 10 years respectively (Figure 1 ).

Characteristics used for multiple regression analyses to correlate with biochemical failure were: clinical T-classification, Gleason score, pretreatment PSA, age, brachytherapy dose level, prostate volume and hormonal ablative treatment.

The multivariate Cox regression analyses identified, Gleason score as independent prognostic factors for biochemical failure.

The 10-year actuarial biochemical control stratified by Gleason score was $85 \%, 72 \%$ and $74 \%$ for patients with Gleason score of $\leq 6,7$ and $>7$, respectively $(P=0.039)$ (Figure 2).

The 10 -year actuarial biochemical control was $89 \%$ for patients with two intermediate risk criteria, $80 \%$ with one high risk criteria and $72 \%$ for patients with 2-3 high risk criteria $(P=0.04)$ (Figure 3$)$.

The 10-year actuarial biochemical control in patients classified as high risk only by Gleason and/or PSA, no by T-stage, was $86 \%$ for patients with two intermediate risk criteria, $73 \%$ with one high risk criteria and $71 \%$ for patients with 2 high risk criteria $(P=0.45)$ (Figure 4).

Mean age was 67 years (range 49-78). The actuarial analysis of biochemical control at ages less than 60,60 to 69 and greater than 69 years demonstrated no significant difference. As younger and older patients benefited equally $(\mathrm{P}=0.148)$.

The actuarial biochemical control was $80 \%$ and $74 \%$ (P $=0.173)$, in patients who received hormones and in those who did not.

All other variables (pretreatment PSA $\mathrm{P}=0.898, \mathrm{~T}$ classification $(\mathrm{p}=0.273)$, brachytherapy dose level $\mathrm{P}=$ 0.492 , and volume $P=0.615$ ) were statistical no significant for biochemical failure.

Metastatic disease developed in 42 patients and the distant metastatic rate at 10 -years was $14 \%$. Multivariate analysis showed that Gleason score was the only factor to significantly affect distant metastases, with 10 -year rates of $7 \%, 21 \%$ and $17 \%$ for scores of $\leq 6,7$ and $>7$, respectively $(\mathrm{P}=0.014)$. 


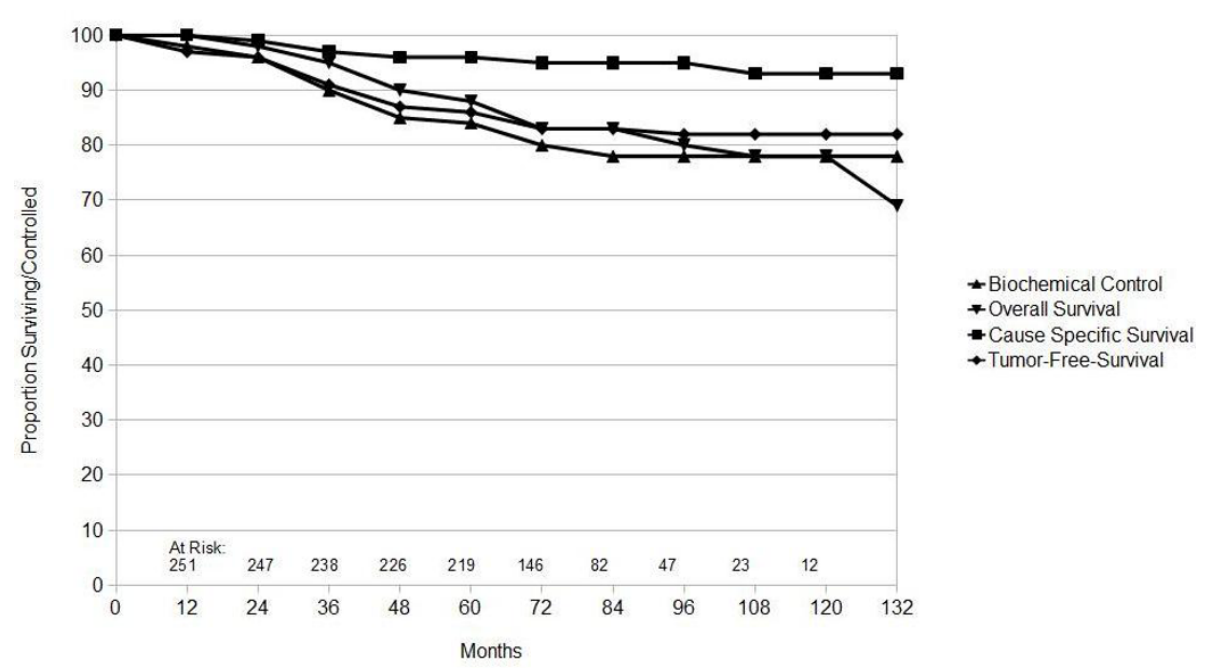

Figure 1 Actuarial analysis of all 252 patients for Cause specific survival, tumor-free survival, biochemical control and overall survival.

The 10-year actuarial tumour-free survival (TFS) was $90 \%, 73 \%$ and $78 \%$ for patients with Gleason scores of $\leq$ 6,7 and $>7$, respectively $(\mathrm{P}=0.020)$.

\section{Acute and chronic urinary toxicity}

Moderate increase in urinary frequency and tract pain (urethritis) occurred at the end of the treatment. At 6 month genitourinary grade I toxicity was $6 \%$ (increase in urinary frequency and dysuria. Moderate symptoms). Acute grade 1 urinary retention requiring a temporary post-implant bladder catheter was seen in $4(1.6 \%)$ patients.

Grade 4 or 5 late toxicity was not detected in any patients. Thirteen patients (5\%) showed, increase in urinary frequency and dysuria at 12 month.
A urethral stricture after treatment was observed in seven patients $(2.7 \%)$. Four patients required intermittent bladder catheterization and endoscopic urethrotomy was required in three patients.

No patients reported incontinence after treatment.

\section{Gastrointestinal toxicity}

Acute gastrointestinal toxicity grade II was 3\%, consisting in increase of 4-6 stools per day over baseline and mucus in stool, no patients developed grade 3 toxicity. At six months gastrointestinal grade II toxicity was $1.6 \%$.

At 12 months $99 \%$ of patients reported no change in bowel function. Intermittent rectal bleeding without systemic repercussions was reported in 5 patients $(2 \%)$. No patients with perineal pain were reported.

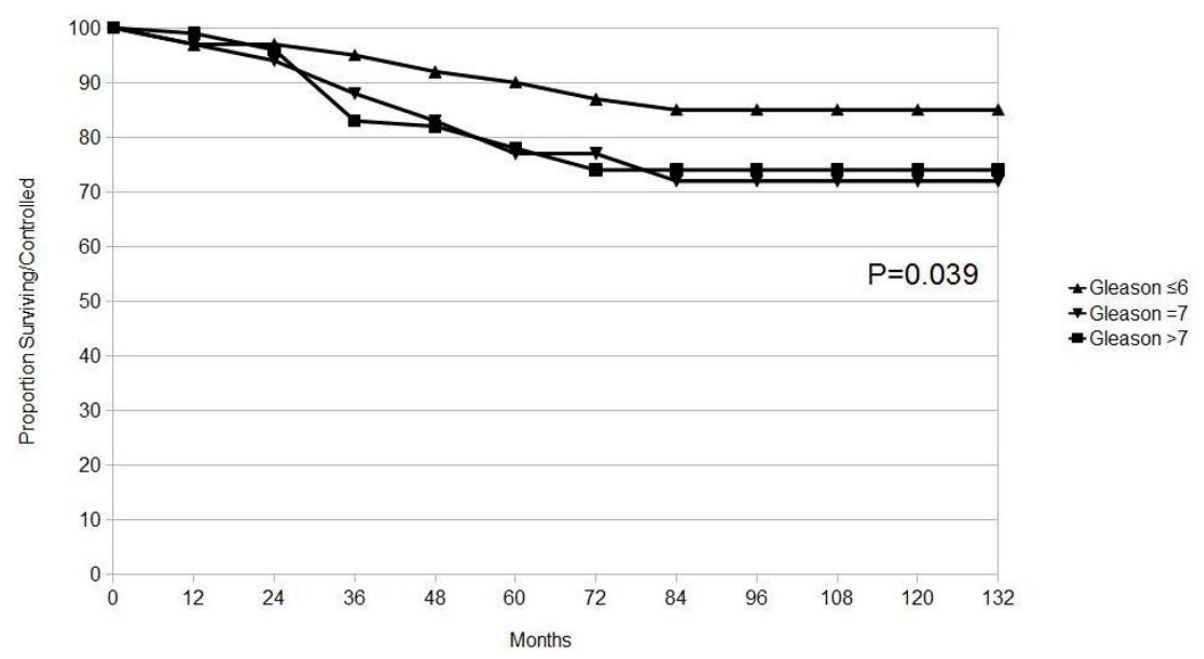

Figure 2 Actuarial analysis of biochemical control by Gleason score. P value generated from Log-Rank. 


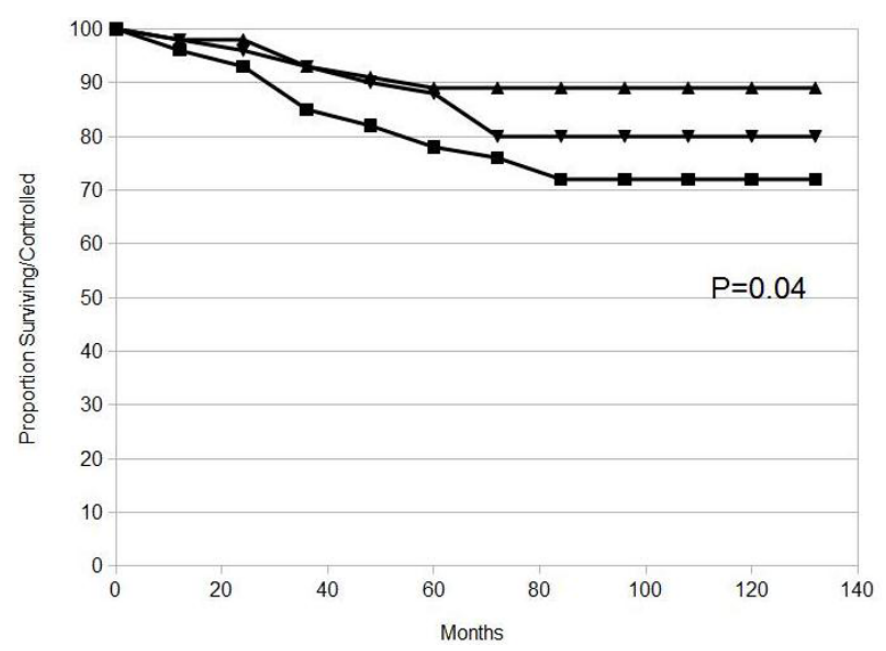

Figure 3 Actuarial analysis of biochemical control by poor prognostic factors groups. P value generated from Log-Rank.

\section{Sexual function}

Of the 131 (52\%) patients who were potent preoperatively and hormonal manipulation was not used, $72 \%$ were potent postoperatively but only $68 \%$ the patients were potent when hormonal manipulation was used. Potency was defined as the ability to achieve an erection that was sufficient for intercourse.

\section{Correlation between post implant dosimetry and toxicity} Characteristics used for multiple logistic-regression analyses which correlates probability of acute and chronic urinary toxicity were: pre-treatment prostate volume, year of implant, number of needles implanted, brachytherapy dose, hormonal ablative treatment, maximum urethral dose.

The multivariate logistic-regression analyses showed than patients with high urethral dose after established brachytherapy technique are more likely to suffer acute urethritis $(\mathrm{P}=0.018)$.

\section{Discussion}

The importance of dose escalation, has been well documented, high radiation doses improve biochemical and clinical results for prostate cancer patients [4-7]. Kuban et al. reported an improvement in biochemical control of $78 \%$ for doses of 78 Gy, vs $59 \%$ for the 70 Gy arm [13].

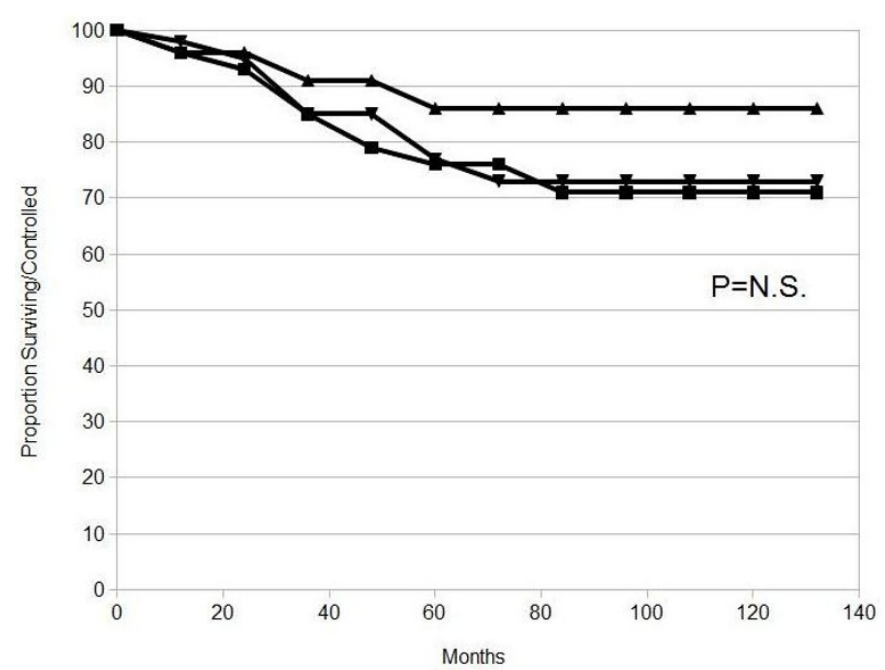

$\$ 2$ Intermediate Risk Criteria

$\rightarrow 1$ High Risk Criteria

$\rightarrow 2$ High Risk Criteria

Figure 4 Actuarial analysis of biochemical control by poor prognostic factors groups in patients classified as high risk only by Gleason and/or PSA, no by T-stage. P value generated from Log-Rank. 
The combination of EBRT and HDR brachytherapy allows the delivery of very high biologic equivalent doses to the prostate not achievable by intensity modulated treatments (IMRT) with image guided adaptive radiotherapy (IGART) techniques.

Based on these principles, since June 1998 we have been performing High dose rate brachytherapy boost for prostate cancer.

The high risk group in our report represented 100\% of the patient population and the results of this combined therapy (EBRT + HDR boost) at 10-years are promising, with a biochemical control rate of $78 \%$, cause specific survival of $93 \%$, overall survival of $78 \%$, and freedom from distant metastases of $86 \%$. The 10 -year actuarial biochemical control in patients classified as high risk only by Gleason and/or PSA, no by T-stage, was $86 \%$ for patients with two intermediate risk criteria, 73\% with one high risk criteria and $71 \%$ for patients with 2 high risk criteria.

The results presented here for high risk group of patients, are superior to the series on radical prostatectomy and standard radiotherapy therapies published in the literature.

The Memorial Sloan Kettering reported in unfavorable risk cases 5-year PSA relapse-free survival rate for 81 Gy the $67 \%$ versus $43 \%$ for 75.6 Gy and $21 \%$ for 64.8 to 70.2 Gy [14].

Hanks et al. observed that patients with unfavorable disease (Gleason $\geq 8$ and PSA $\geq 20 \mathrm{ng} / \mathrm{ml}$ ) treated with three-dimensional conformal radiation therapy (3D CRT) a dose of 76 Gy achieved only $26 \%$ in the 5 -year PSA relapse-free survival rate [15]. Dearnaley et al., Sathya et al. and Zietman et al. reported similar results [16-18].

Our study shows an advantage to high-dose over conventional-dose conformal radiation in terms of freedom from biochemical failure for men with high risk prostate cancer.

Similar observations were reported by other institutions using conformal high dose rate brachytherapy. Martinez et al. [19] reported 5-year actuarial biochemical control rate of $85 \%$ for patients with 1 poor prognostic factor, $75 \%$ for 2 and $50 \%$ for all 3 . Galalae et al. reported 8-year bNED survival rate (free of clinical or biochemical evidence of disease) in the high-risk prognostic group of 64\% [20]. Dattoli et al. [21] and Mate et al. [22] reported similar results. A recent report Stock et al. reported 8-year actuarial biochemical control rate of $73 \%$ for patients with Gleason score 8-10 prostate cancer [23].

On the other hand, surgery is not the best treatment for high risk patients. Catalona et al. [24] Studied 3478 men with tumors of clinical stages T1-T3 No M0 followed for an average of 65 months after radical retropubic prostatectomy. Actuarial 10-year biochemical progression-free probabilities were $59 \%$ for cT2b-c, $15 \%$ for cT3 disease and 50\% for Gleason sum $4+3$ and 32\% for Gleason 8-10 disease. Actuarial 10-year biochemical progression-free probabilities were $49 \%$ for PSA greater than $10 \mathrm{ng} / \mathrm{ml}$.

The Johns Hopkins group reported similar results $[25,26]$. Kermen and Miles [27] reported a 5-year bNED rate of $54 \%$ after radical prostatectomy. Surgery results of Multi-institutional pooled analysis in men with locally advanced prostate cancer [2] published a 2.2-year of biochemical control rate of $16 \%$.

The Memorial Sloan Kettering Center group analyzed the oncologic outcome after laparoscopy radical prostatectomy, 8-year probably of freedom from progression for high risk cancer was 53\% [28].

In patients with high risk prostate cancer, the 10-year biochemical control was $16-54 \%$ for prostatectomy treated patients compared to $86-71 \%$ for our combined conformal EBRT with HDR boost.

Despite the wide diffusion of laparoscopic radical prostatectomy and robot-assisted laparoscopic radical prostatectomy, only few studies comparing the results of these techniques with the retropubic radical prostatectomy. The systematic review of the literature performed by Ficarra et al. [29], were not sufficient to prove the superiority of any surgical approach in terms of functional and oncologic outcomes.

Gleason score was in our paper the most significant predictor of biochemical failure and developing distant metastases. Other groups reported similar results $[19,21,23]$.

Hormonal ablative treatment did not improved the outcome in the present analysis, this corroborate the findings of previous studies [21,30,31]. All prospective, randomized trials show a positive outcome of adding hormonal therapy [32-34] but these studies were done with low radiation doses (68-70 Gy), the BED equivalent of 70 Gy was 129 Gy, much lower than BED in our series (BED dose ranged from 292 Gy to 366 Gy).

The low toxicity observed in our series, despite the high radiation doses delivered, was the result of carefully executed real-time brachytherapy technique [35]. Urinary and Gastrointestinal complications rates were in concordance with the experience of other institutions using conformal high doses rate brachytherapy [19-22] and favourably with other 3-D conformal radiotherapy escalating series $[14,15]$.

The cause specific survival at 10 -year of $93 \%$ and biochemical control of $78 \%$ demonstrate the effectiveness of the described radiotherapy regime and the high curative potential of this therapy protocol. 


\section{Conclusions}

The present studies shows that when men with high risk clinically localized prostate cancer are treated with EBRT interdigitated with two HDR Ir-192 brachytherapy boost, allows us to administer the highest possible dose to the prostate and the lowest dose to the surrounding healthy structures, achieved excellent results in terms of local and biochemical control, decrease the toxicity and the overall treatment time by at least 3 weeks compared to 3-D conformal radiation therapy and intensity modulated.

In summary this treatment regimen is a safe and very effective for patients with high risk localized prostate cancer and represents a considerable improvement over standard surgical and radiotherapy modalities.

\section{Abbreviations}

AJCC: American Joint Committee on Cancer; BED: Biologically effective dose; BTm: Brachytherapy; bNED: No clinical and biochemical evidence of disease; CTV: Clinical target volume; CSS: Cancer specific survival; CT: Computed tomography; D90: The dose that covers $90 \%$ volume of CTV; 3D CRT: Threedimensional conformal radiation therapy; EBRT: External beam radiotherapy; GU: Genitourinary; HDR: High dose rate; IMRT: Intensity modulated radiotherapy; IGART: Image guided adaptive radiotherapy; PSA: Serum prostate-specific antigen; PTV: Planning target volume; CTAE v4.02: Common Toxicity Criteria for Adverse Event, Version 4.0 by the National Cancer Institute; SPSS: Statistical analysis SPSS; SD: Standard desviations; TFS: Tumour-free survival; TRUS: The trans-rectal ultrasound; V100, V90, V150 and V200: (\% volume of CTV receiving 100\% of prescription dose).

\section{Author details}

${ }^{1}$ Department of Radiation Oncology, Hospital Universitario Central de Asturias, Oviedo, Spain. ${ }^{2}$ Department of Radiation Physics, Hospital Universitario Central de Asturias, Oviedo, Spain. ${ }^{3}$ Department of Radiation Oncology, Hospital Central de Asturias, C/Julian Claveria s/n, Oviedo 33006, (Asturias), Spain.

\section{Authors' contributions}

P.JP: conception and design. LM acquisition of data. JF analysis of data. HG revising references. IJ alignment and drafted the manuscript. EA acquisition of data. All authors read and approved the final manuscript.

\section{Competing interests}

The authors declare that they have no competing interests.

Received: 18 December 2011 Accepted: 7 March 2012

Published: 7 March 2012

\section{References}

1. Morgan WR, Bergstralh EJ, Zincke $\mathrm{H}$ : Long-term evaluation of radical prostatectomy as treatment for clinical stage C (T3) prostate cancer. Urology 1993, 41:113-120.

2. Gerber GS, Thisted RA, Chodak GW, et al: Results of radical prostatectomy in men with locally advanced prostate cancer: multi-institutional pooled analysis. Eur Urol 1997, 32:385-390.

3. Hanks GE, Diamond JJ, Kual JM, et al: 17 ten-year follow-up of 682 patients treated for prostate cancer with radiation therapy in the United States. Int J Radiat Oncol Biol Phys 1987, 13(4):499-505.

4. Pollack A, Zagars GK: External beam radiotherapy dose-response of prostate cancer. Int J Radiat Oncol Biol Phys 1997, 39(5):1011-1018.

5. Pollack A, Zagars GK, Smith LG, et al: Preliminary results of a randomized radiotherapy dose escalation study comparing 70 Gy to 78 Gy for prostate cancer. J Clin Oncol 2000, 18(23):3904-3911.
6. Hanks EG, Hanlon AL, Pinover WH, et al: Dose selection for prostate cancer patients based on dose comparison and dose response studies. Int J Radiot Oncol Biol Phys 2000, 46(4):823-832.

7. Hanks GE, Martz KL, Diamond Jj: The effect of dose on local of prostate cancer. Int J Radiat Oncol Biol Phys 1988, 15(6):1299-1305.

8. Brenner DJ, Martinez AA, Edmundson GK, et al: Direct evidence that prostate tumors show a high sensitivity to fractionation (low $\alpha / \beta$ ), similar to late-responding normal tissue. Int J Radiat Oncol Biol Phys 2002, 52(1):6-13.

9. American Joint Committee on cancer: Prostate. In AJCC Cancer Staging Manual.. 4 edition. Edited by: Beahrs OH, Henson DE, Htter RVP, Kennedy BJ. Philadelphia: Lippincott-Raven; 1992:181-186.

10. Zelefsky MJ, Leibel SA, Gaudin PB, et al: Dose escalation with threedimensional conformal radiation therapy affects the outcome in prostate cancer. Int J Radiat Oncol Biol Phys 1998, 41:491-500.

11. Roach M, Hanks $G$, Thames $H$, et al: Defining biochemical failure following radiotherapy with or without hormonal Therapy in men with clinically localized prostate cancer: Recommendations of the RTOG-ASTRO Phoenix Consensus Conference. Int J Radiat Oncol Biol Phys 2006, 65:965-974.

12. Cox DR: Regression models and life-tables. J R Stat Soc B 1972, 34:187-220.

13. Kuban DA, Tucker SL, Dong L, et al: Long-term results of the M.D. Anderson randomized dose-escalation trial for prostate cancer. Int J Radiat Oncol Biol Phys 2008, 70:67-74.

14. Zelefsky MJ, Fuks Z, Hunt M, et al: Margie Hunt et al. High dose radiation delivered by intensity modulated conformal radiotherapy improves the outcome of localized prostate cancer. J Urology 2001, 166:876-881.

15. Hanks GE, Hanlon AL, Pinover WH, et al: Dose selection for prostate cancer patients based on dose comparison and dose response studies. Int J Radiat Oncol Biol Phys 2000, 46(4):823-832.

16. Dearnaley DP, Hall E, Lawrence D, et al: Phase III pilot study of dose escalation using conformal radiotherapy in prostate cancer: PSA control and side effects. Br J Cancer 2005, 92:488-498.

17. Sathya JR, Davis IR, Julian JA, et al: Randomized trial comparing iridium implant plus external-beam radiation therapy with external-beam radiation therapy alone in node-negative locally advanced cancer of the prostate. J Clin Oncol 2005, 23:1192-1199.

18. Zietman AL, DeSilvio ML, Slater JD, et al: Comparison of conventional-dose vs high-dose conformal radiation therapy in clinically localized adenocarcinoma of the prostate: a randomized controlled trial. JAMA 2005, 294:1233-1239.

19. Martinez A, Gonzalez J, Spencer W, et al: Conformal High dose rate brachytherapy improves biochemical control and cause specific survival in patients with prostate cancer and poor prognostic factors. J Urology 2003, 169:974-980

20. Galalae RM, Kovács G, Schultze J, et al: Long-term outcome after elective irradiation of the pelvic lymphatics and local dose escalation using High-dose- rate brachytherapy for locally advanced prostate cancer. Int J Radiat Oncol Biol Phys 2002, 52:81-90.

21. Dattoli $M$, Wallner $K$, True $L$, et al: Long-term outcome after treatment with brachytherapy and supplemental conformal radiation for prostate cancer patients having intermediate and high-risk features. Cancer 2007, 110(3):551-555.

22. Mate TP, Gottesman JE, Hatton J, et al: High dose-rate after loading 192 iridium prostate brachytherapy: Feasibility report. Int J Radiat Oncol Biol Phys 1998, 41:525-533.

23. Stock RG, Cesareti JA, Hall SJ, Stone NN: Outcome for patients with highgrade prostate cancer treated with a combination of brachytherapy, external beam radiotherapy and hormonal therapy. BJU Int 2009, 104:1631-1636.

24. Roehl KA, Han M, Ramos CG, et al: Cancer progression and survival rates following anatomical radical retropubic prostatectomy in 3478 consecutive patients: Long-term results. J Urol 2004, 172:910-914.

25. Han M, Partin AW, Zahurak M, et al: Biochemical (prostate specific antigen) recurrence probability following radical prostatectomy for clinically localized prostate cancer. J Urol 2003, 169:517-523.

26. Partin AW, Pound CR, Clemens JQ, et al: Serum PSA after anatomic radical prostatectomy. The Johns Hopkins Experience after 10 years. Urol Clin N Am 1993, 20:713-725. 
27. Kernen KM, Miles BJ: Prostate cancer treatment outcomes after radical prostatectomy: The Baylor experience (Abstr). Radiother Oncol 2000, 55(Suppl 1):7-8

28. Walz J, Joniau S, Chun FK, et al: Pathological results and rates of treatment failure in high-risk prostate cancer patients after radical prostatectomy. BJU Int 2010, 107:765-770.

29. Ficarra V, Novara G, Artibani W, et al: Retropubic laparoscopy, and robotassisted radical prostatectomy: a systematic review and cumulative analysis of comparative studies. Eur Urol 2009, 55:1037-1063.

30. Merrick GS, Butler WM, Galbreath RW, et al: Does hormonal manipulation in conjunction with permanent interstitial brachytherapy, with or without supplemental external beam irradiation, improve the biochemical outcome for men with intermediate or high-risk features? BJU Int 2003, 91(1):23-29.

31. Martinez A, Galalae R, Gonzalez J, et al: No apparent benefit at 5 years from a course of neoadjuvant/concurrent androgen deprivation for patients with prostate cancer treated with high total radiation dose. J Urol 2003, 170(6):2296-2301.

32. Bolla $M$, Collette L, Blank L, et al: Long-term results with immediate androgen suppression and external irradiation in patients with locally advanced prostate cancer (an EORTC study): a phase III randomized trial. Lancet 2002, 360:103-106.

33. Denham JW, Steigler A, Lamb DS, et al: Shortterm androgen deprivation and radiotherapy for locally advanced prostate cancer: results from the Trans-Tasman Radiation Oncology Group 96.01 randomized controlled trial. Lancet Oncol 2005, 6(11):841-850.

34. Pilepich MV, Winter K, Lawton CA, et al: Androgen suppression adjuvant to definitive radiotherapy in prostate carcinoma-long term results of Phase III RTOG 85-31. Int J Radiat Oncol Biol Phys 2005, 61(5):1285-1290,

35. Prada PJ, Fernández J, Martínez A, et al: Transperineal injection of hyaluronic acid in the anterior peri-rectal fat to decease rectal toxicity from radiation delivered with intensity modulated brachytherapy or EBRT for prostate cancer patients. Int I Radiat Oncol Biol Phys 2007, 69(1):95-102.

doi:10.1186/1748-717X-7-31

Cite this article as: Prada et al: Long-term biochemical results after high-dose-rate intensity modulated brachytherapy with external beam radiotherapy for high risk prostate cancer. Radiation Oncology 2012 7:31.

\section{Submit your next manuscript to BioMed Central and take full advantage of:}

- Convenient online submission

- Thorough peer review

- No space constraints or color figure charges

- Immediate publication on acceptance

- Inclusion in PubMed, CAS, Scopus and Google Scholar

- Research which is freely available for redistribution 